\title{
Proteolysis Targeting Chimeras With Reduced Off-targets
}

Tuan M. Nguyen ${ }^{1,2,3, \S}$, Arghya Deb ${ }^{1,2, \S}$, Praveen Kokkonda ${ }^{1,2, \S}$, Vedagopuram Sreekanth ${ }^{1,2,3, \S}$, Praveen K. Tiwari ${ }^{1,2,3}$, Veronika Shoba ${ }^{1,2}$, Sophia Lai ${ }^{1,2,5}$, Santosh K. Chaudhary ${ }^{1,2}$, Jaron A. M. Mercer ${ }^{4,5,6}$, Max Jan $^{7,8,9}$, David R. Liü ${ }^{4,5,6}$, Benjamin L. Ebert ${ }^{7,8,9}$, Amit Choudhary ${ }^{1,2,3, *}$

${ }^{1}$ Chemical Biology and Therapeutics Science, Broad Institute of MIT and Harvard, Cambridge, MA 02142, USA 2Department of Medicine, Harvard Medical School, Boston, MA 02115, USA

${ }^{3}$ Divisions of Renal Medicine and Engineering, Brigham and Women's Hospital, Boston, MA 02115, USA

${ }^{4}$ Merkin Institute of Transformative Technologies in Healthcare, Broad Institute of MIT and Harvard, Cambridge, MA 02142, USA

${ }^{5}$ Department of Chemistry and Chemical Biology, Harvard University, Cambridge, MA 02138, USA

${ }^{6}$ Howard Hughes Medical Institute, Harvard University, Cambridge, MA 02138, USA

${ }^{7}$ Department of Medical Oncology, Dana-Farber Cancer Institute, Boston, MA 02115, USA

${ }^{8}$ Broad Institute of MIT and Harvard, Cambridge, MA 02142, USA

${ }^{9}$ Howard Hughes Medical Institute, Boston, MA 02115, USA

§These authors contributed equally to this work

*Correspondence: achoudhary@bwh.harvard.edu (A.C.)

\author{
Amit Choudhary \\ Chemical Biology and Therapeutics Science \\ Broad Institute of MIT and Harvard \\ 415 Main Street, Rm 3012 \\ Cambridge, MA 02142 \\ Phone: (617) 714-7445 \\ Fax: (617) 715-8969 \\ Email: achoudhary@bwh.harvard.edu
}




\section{ABSTRACT}

Proteolysis Targeting Chimeras (PROTACs), a class of heterobifunctional molecules that recruit target proteins to E3 ligases, have gained traction for targeted protein degradation. However, pomalidomide, a widely used E3 ligase recruiter in PROTACs, can independently degrade other targets, such as zinc-finger (ZF) proteins, that hold key functions in normal development and disease progression. This off-target degradation of pomalidomidebased PROTACs raises concerns about their therapeutic applicability and long-term side effects. Therefore, there is a crucial need to develop rules for PROTAC design that minimize off-target degradation. In this study, we developed a high-throughput platform that interrogates the off-target degradation of ZF domains and discovered, using this platform, that PROTACs with the current design paradigm induce significant degradation of several ZF proteins. To identify new rules for PROTAC design, we generated a rationalized library of pomalidomide analogs with distinct exit vector modifications on the C4 and C5 positions of the phthalimide ring and profiled their propensities for ZF protein degradation. We found that modifications on the C5 position with nucleophilic aromatic substitution $\left(S_{N} A r\right)$ reduce off-target ZF degradation. We applied our newfound design principles on a previously developed ALK oncoprotein-targeting PROTAC and generated PROTACs with enhanced potency and minimal off-target degradation. We envision the reported off-target profiling platform and pomalidomide analogs will find utility in design of specific PROTACs. 


\section{INTRODUCTION}

Immunomodulatory imide drugs (IMiD)-based molecular glues (e.g., pomalidomide) induce proximity between cereblon (CRBN), the substrate receptor for an E3 ubiquitin ligase, and proteins with Zn-finger (ZF) motifs to trigger ubiquitination and degradation of the latter. ${ }^{1-6}$ Pomalidomide is often appended to target protein binders to generate CRBN-based Proteolysis Targeting Chimeras (PROTACs) that induce proximity-mediated target protein degradation..$^{7-9}$ However, these pomalidomide-based PROTACs can also recruit other proteins with or without ZF motifs that serve key biological functions in normal development and disease progression. ${ }^{10-14}$ For example, tissue-specific deletion of pomalidomide-degradable ZF protein, ZFP91, in regulatory T cells (Tregs) leads to Treg dysfunction and increases the severity of inflammation-driven colorectal cancer. ${ }^{15}$ Furthermore, there are numerous other proteins with important roles in cellular function, such as transcription factors, that also harbor ZF domains. ${ }^{16-17}$ The off-target degradation of these key ZF-containing proteins may have long-term implications such as the development of new cancers, dysregulation of lymphocyte development, and teratogenic effects. ${ }^{18-22}$ The ability of pomalidomide to degrade other proteins in a PROTAC-independent manner raises concerns about the precariousness of off-target ubiquitination and degradation of these compounds, several of which are already in clinical trials. ${ }^{23-24}$ Thus, there is an urgent need for robust, sensitive, and high-throughput methods that can determine off-target degradation in such PROTACs. ${ }^{25}$

Currently, off-target degradation can be assessed by mass-spectrometry-based methods ${ }^{26-29}$ that detect protein levels, but these techniques lack sensitivity for low abundant proteins.$^{30}$ In addition to expense, the implementation of mass spectrometry is technically challenging when analyses include profiling the off-target degradation affected by specific PROTACs across multiple tissue types for tissue-specific expression of lineage-specific proteins. ${ }^{31}$ These analyses are further complicated by the need to perform these assessments across different levels of PROTAC dosing.

Herein, we report the development of a sensitive, robust, and high-throughput imaging platform that profiles the off-target degradation propensities of ZF domains by measuring the decrease in fluorescence of a panel of GFPtagged various ZF domains upon compound treatment. ${ }^{32}$ With this platform, we profiled off-target activities of 
literature-reported PROTACs with different exit vectors. Surprisingly, nearly all the profiled PROTACs exhibited significant off-target degradation of ZF domains. To reduce this, we rationally designed and generated eighty pomalidomide analogs and profiled them for off-target activity. We discovered that substitution at specific positions (C5) on pomalidomide significantly reduced degradation propensities. With these findings, we generated new PROTACs that target anaplastic lymphoma kinase (ALK) with reduced off-target ZF degradation and enhanced on-target potencies. Overall, we developed an off-target profiling platform and used that platform to identify pomalidomide analogs with lower off-target propensity and then deployed such analog to generate a specific PROTAC for ALK.

\section{RESULTS AND DISCUSSION}

Development and validation of an off-target profiling platform for PROTACs. To profile the ZF degradation propensity of pomalidomide and its analogs, we first developed an automated imaging assay (Figure 1A). For this platform, we selected 23-amino-acid ZF degrons of 11 ZF proteins that are reportedly degraded by pomalidomide and 3 ZFs that are not (Supplementary Table S1). ${ }^{32}$ We inserted these ZF degrons into a lentiviral degradation reporter vector (cilantro 2$)^{32}$ to compare the fluorescence of ZF-tagged enhanced green fluorescent protein (eGFP) to untagged mCherry (Figure 1A). With this assay, wherein compounds are tested against the 14 stable U2OS cell lines containing our tagged ZF-proteins, we reliably detected dose-dependent degradation of ZF degron-tagged eGFP by pomalidomide with a robust readout ( $Z^{\prime}=0.8$ ) (Figure S1). As expected, pomalidomide degraded all 12 ZF degrons that are sensitive to it in a dose-dependent manner that ranged from $4.3 \mathrm{nM}$ to $6 \mu \mathrm{M}$ (Figure 1A). Unlike mass spectrometry, this reporter-based method is not limited by cell-type-specific expression levels of analyte proteins nor by the accessibility to ZFs in the context of full-length proteins that are engaged in protein complexes. Thus, this method may have enhanced sensitivity over mass spectrometry-based methods for the detection of pomalidomide-sensitive ZF protein degradation.

With this assay in hand, we profiled the off-target activity of 9 reported PROTACs with varying exit vectors from pomalidomide end and linker lengths (Figure 1B, Figure S2). We observed significant degradation of many ZFdomains with almost all the 9 PROTACs (Figure 1B). Notably, PROTACs with common exit vectors, such as arylamine, -ether, -carbon, and -amide, generally had greater ZF degradation capabilities in similar fashion to 
bioRxiv preprint doi: https://doi.org/10.1101/2021.11.18.468552; this version posted November 20, 2021. The copyright holder for this preprint (which was not certified by peer review) is the author/funder, who has granted bioRxiv a license to display the preprint in perpetuity. It is made available under aCC-BY-NC-ND 4.0 International license.

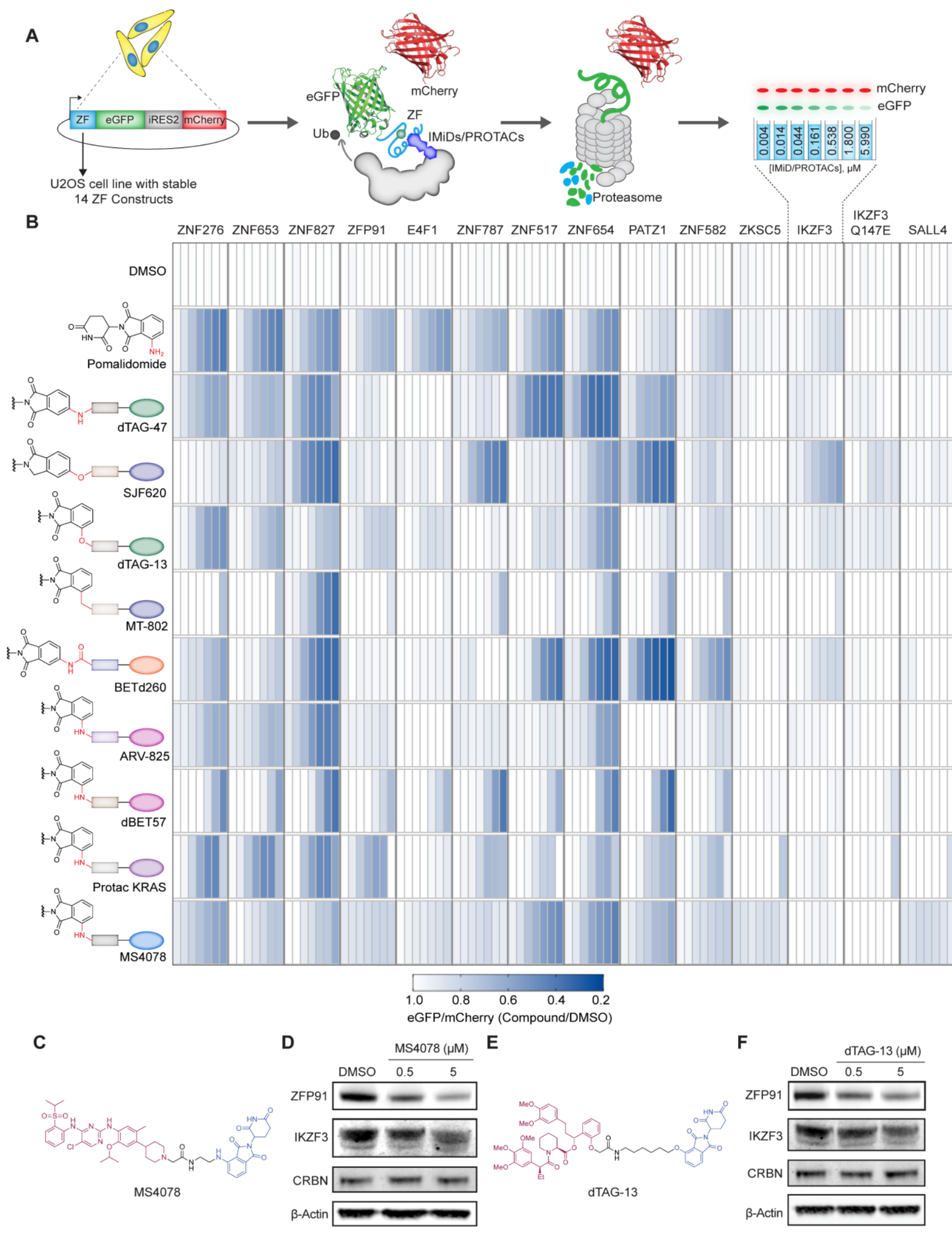

Figure 1. Development of a high-throughput assay for the evaluation of off-target ZF degradation of pomalidomide-based PROTACs. (A) Schema of the automated imaging screen for the degradation of ZF 
degrons by pomalidomide analogs and pomalidomide-based PROTACs. Briefly, U2OS cells stably expressing 14 ZF degrons fused to eGFP were treated with PROTACs followed by imaging to assess ZF degradation. (B) Degradation of validated and pomalidomide-sensitive ZF degrons inside cells by reported PROTACs in a dosing range of $4.3 \mathrm{nM}$ to $6 \mu \mathrm{M}$. (C-D) Immunoblots demonstrating off-target degradation of endogenous $\mathrm{ZF}$ proteins ZFP91 and IKZF3 by (C) MS4078 (ALK PROTAC) and (D) dTAG-13 (FKBP12 F36V PROTAC) in a dose-dependent manner in Jurkat cells.

pomalidomide (Figure 1B and 3). Our assay also confirmed the off-target degradation of endogenous ZF proteins such as ZFP91 and IKZF3 by reported PROTACs MS4078 (ALK PROTAC, Figure 1C) ${ }^{33}$ and dTAG-13 (FKBP12 ${ }^{\mathrm{F} 36 \mathrm{~V}}$ PROTAC, Figure 1E) ${ }^{34}$, as shown in immunoblots (Figure 1D, 1F, and S3). These data suggest that PROTACs with linkers on $4^{\text {th }}$ position of the aryl ring induce significant degradation of off-target ZF proteins.

Next, we queried the influence of exit vectors on pomalidomide-based PROTACs and the degradation of ZFs. Towards this goal, we analyzed changes in endogenous ZF proteins from 124 proteomics datasets that were generated for cells treated with pomalidomide-based PROTACs. ${ }^{31}$ In Figure S4, we show the relative abundance of proteins that contained the ZF motif as previously described ${ }^{32}$ and were detectable in at least 1 proteomics dataset (i.e., 284 ZF proteins). We computed a ZF degradation score for every PROTAC dataset by taking the sum of ZF protein abundance. Analyzing the degradation score distribution confirmed that PROTACs had significant ZF protein degradation activity for amino acetamide and arylamine, -ether, and -carbon exit vectors (Figure S4). Both the analysis of these proteomic datasets of endogenous proteins and our image-based profiling assay suggests that the flexible exit vectors on position 4 of the aryl ring induce more overall ZF degradation (Figure 1B and S4). The agreement between our automated imaging assay and proteomics suggests that we can apply our methodology in a high-throughput manner to identify new pomalidomide-based PROTACs and pomalidomide analogs that confer minimal ZF degradation. Furthermore, we can determine a new set of rules for PROTAC development to minimize off-target degradation of endogenous ZF proteins.

Generation of a library of rationally designed pomalidomide analogs. We next endeavored to create a library of rationally designed pomalidomide analogs that can be applied towards the systematic design of pomalidomide-based PROTACs with minimal off-target ZF degradation. We gained structural insight from the crystal structure of the DDB1-CRBN-pomalidomide complex bound to transcription factor IKZF1 (PDB: 6HOF). ${ }^{32}$ In the crystal structure, the glutarimide ring of pomalidomide is deeply buried inside CRBN while the phthalimide ring 
is accessible for modification. Q146 of IKZF1 forms a water-mediated hydrogen-bonding interaction with the C4 amino group of the compound, while the C5 position is proximal to the ZF domain (Figure $2 \mathrm{~A}$ ). ${ }^{32}$ Mutation of Q146, or equivalent residues, has been reported to abrogate IKZF degradation. ${ }^{32}$ We hypothesized that appropriate substitutions at the C4 and/or C5 positions could disrupt the ternary complex of the ZF domains with CRBN while maintaining its interaction with CRBN through glutarimide ring. To investigate this, we first synthesized a thalidomide analog, 5-aminothalidomide (38, Figure 2B). The treatment of MM1.S cells with this C5-amino analog revealed a decrease in overall degradation potency as compared to pomalidomide (1, Figure $2 \mathrm{~B})$, suggesting that modifications on the C5 position will "bump-off", or eliminate, the endogenous ZFs while preserving the CRBN interaction. Therefore, we generated 80 imide analogs (Figure S5) with varying exit vector modifications on the C4 and C5 positions of the phthalimide ring. The modified IMiD analogs should not affect the recruitment of CRBN for inducing protein degradation since the imide ring does not interact with the ubiquitin ligase (Figure $2 \mathrm{~A}$ and S6A-C).

To rapidly and systematically construct the library of pomalidomide analogs, we leveraged reactions like nucleophilic aromatic substitution $\left(S_{N} A r\right)$, amidation, Suzuki, and Sonogashira cross-couplings with commercially available imide compounds for the facile and scalable incorporation of C4 and C5 substitutions on the phthalimide ring (Figure S5). We synthesized the pomalidomide analogs in pairs at C4 and C5 positions and generated a library of 80 compounds that we categorized into three main synthetic groups: $\mathrm{C}-\mathrm{N}\left(\mathrm{S}_{\mathrm{N}} \mathrm{Ar}\right), \mathrm{N}-\mathrm{C}$ (acylation), and C-C (Suzuki/Sonogashira cross-coupling) (Figure S5). We carried out amidation with a diverse class of acids varying from aliphatics to heterocyclic cores and varied the sizes of the carboxylic acid to range from acetic acid to the largest cubane carboxylic acid. We also employed a number of aliphatic amines with variable sizes for high yielding $S_{N} A r$ reactions with 4- and 5-F thalidomides. For the $S_{N} A r$ library, we incorporated a number of aliphatic amines like $N$-Boc piperazine and $N$-Boc diazaspiro[3.3]heptane, which can subsequently be used for PROTAC synthesis after validation. In addition to these $S_{N} A r$ libraries, we synthesized several pomalidomide analogs with a fluoro substitution at the 6-position of thalidomide as well as a number of compounds with heterocyclic boronic acids and phenylacetylene coupled with the 4- and 5- bromo thalidomides. The physicochemical properties of the overall library encompass a reasonable distribution of drug-like properties, such as partition 
coefficient, or cLogP (-2 to 4), molecular weights (250-550 g/mol), and topological polar surface area (tPSA, 80 to $140 \AA^{2}$ ) (Figure S6D).

Systematic evaluation of ZF protein degradation propensity of pomalidomide analogs. Using our developed off-target profiling platform, we tested the library of pomalidomide analogues to derive rules for the impact of exit vector modifications on pomalidomide and ZF protein degradation. First, we observed that analogs with C5 modifications on the phthalimide ring had reduced ZF degradation relative to identical modifications on the C4 position, particularly for $S_{N} \operatorname{Ar}(C-N)$ analogs (Figure $\left.2 B, S 7 A\right)$. The trend was less significant for modifications made with amidation $(\mathrm{N}-\mathrm{C})$ and Suzuki/Sonogashira cross-couplings $(\mathrm{C}-\mathrm{C})$, partly due to having smaller sets of analogs compared with the $S_{N} A r$ group (Figure S7B-C). Our structure modeling and docking data also suggest that C5 modifications are more likely to create a steric clash between the $S_{N} A r$ exit vectors and the ZF domain than $\mathrm{C} 4$ position modifications (Figure S6A-C). Second, analogs lacking hydrogen-bond (H-bond) donors immediately attached to the phthalimide ring had significantly reduced ZF degradation compared to those with $\mathrm{H}$-bond donors, regardless of the position of the modification relative to the ring (Figure 2E), agreeing with our ZF degradation profiling for reported PROTACs (Figure 1B). The immunoblotting results also agree with the highthroughput imaging data that confirm the C5-C4 position-dependent effect on the degradation of endogenous ZF proteins, ZFP91 and IKZF3 (Figure 2F). Taken together, these data reveal that pomalidomide-based PROTACs with an arylamine exit vector, where $\mathrm{NH}$ - is a $\mathrm{H}$-bond donor, induced greater ZF degradation and suggest that PROTACs without H-bond donors would make the PROTACs with minimal off-target activity. This finding is consistent with published data demonstrating that the ability of pomalidomide to form $\mathrm{H}$-bonds is essential for the ternary complex between ZF and CRBN for subsequent ZF degradation. ${ }^{32}$ We aimed to further minimize off-target ZF degradation in $\mathrm{S}_{N} \mathrm{Ar}$ analogs with C5 position modifications through addition of a fluoro group at the C6 position (Figure S5). The fluoro group reduced ZF degradation for most $\mathrm{S}_{\mathrm{N}} A$ r exit vectors, such as $\mathrm{N}$-acetylpiperazine and morpholine, but not for diazaspiro[3.3]heptane (Figure S57A and S7D). 
bioRxiv preprint doi: https://doi.org/10.1101/2021.11.18.468552; this version posted November 20, 2021. The copyright holder for this preprint (which was not certified by peer review) is the author/funder, who has granted bioRxiv a license to display the preprint in perpetuity. It is made available under aCC-BY-NC-ND 4.0 International license.

A

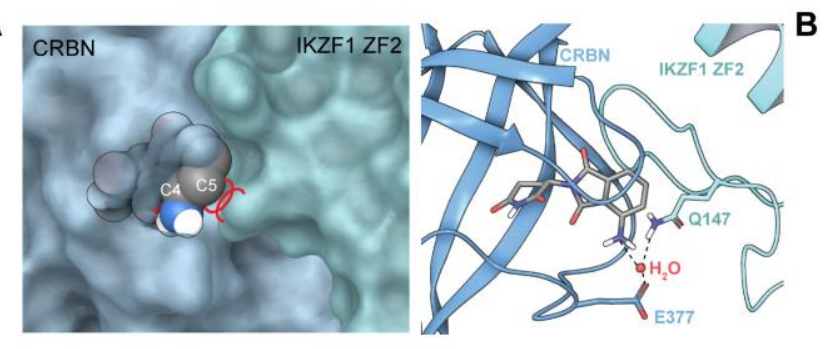

C

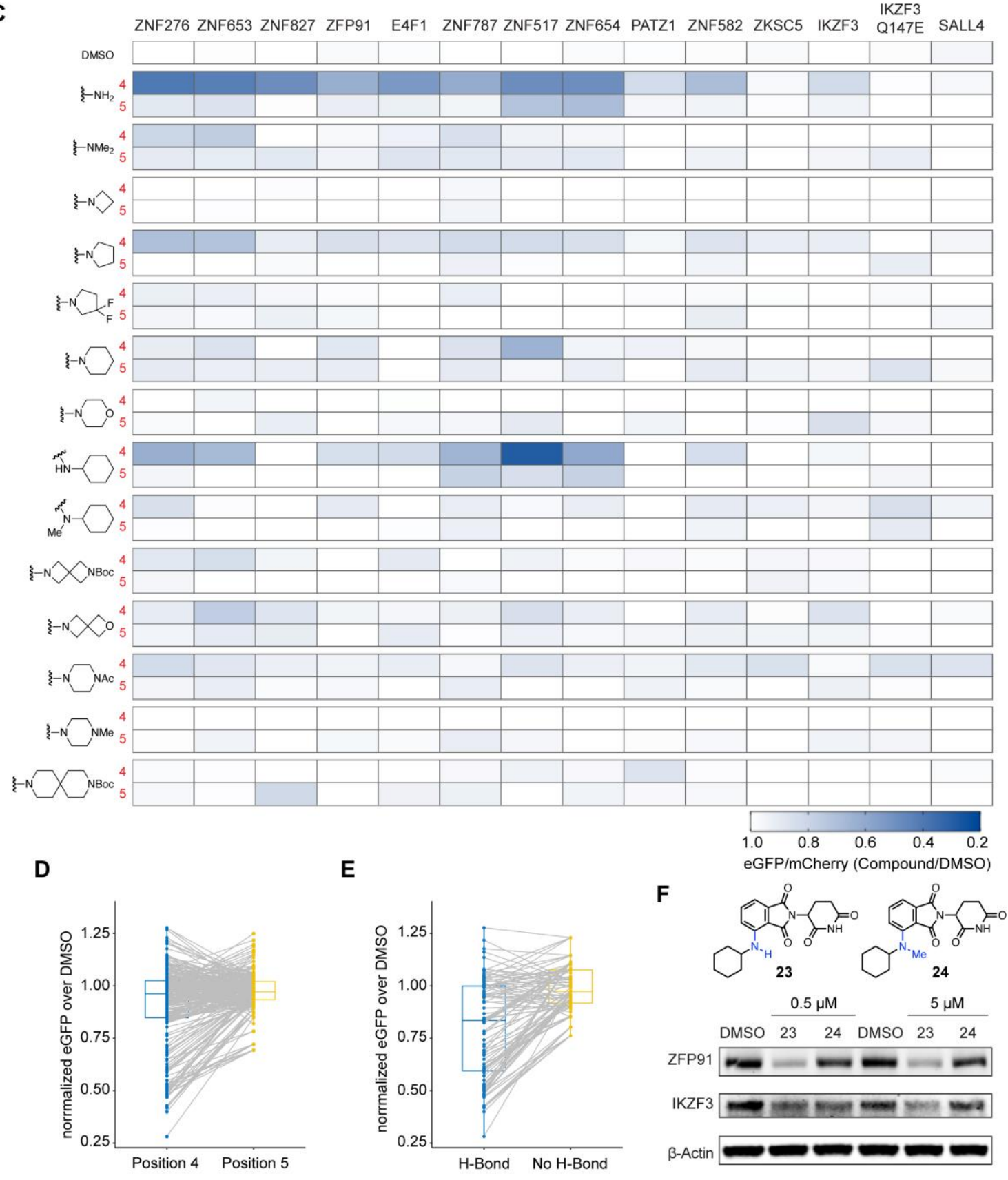

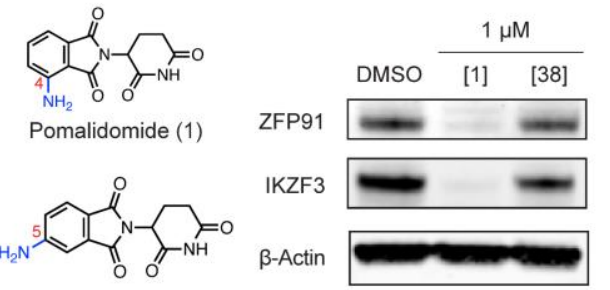

5-Aminothalidomide (38) 
MM1.S cells treated with pairs of imide-based analogs with C4 (pomalidomide) and C5 amino modifications on the phthalimide ring. (C) Degradation of pomalidomide-sensitive ZF degrons inside cells by the $S_{N} A r$ group of pomalidomide analogs arranged in pairs of C4 and C5 modifications on the phthalimide ring. Data for cells treated with $6 \mu \mathrm{M}$ of each compound are shown. (D) Plot showing pair-wise comparison of GFP degradation levels induced by pairs of $S_{N} A r$ pomalidomide analogs with C4 and C5 modifications at the same dose, ranging from $4.3 \mathrm{nM}$ to $20 \mu \mathrm{M}$. (E) Degradation of pomalidomide-sensitive ZF degrons inside cells by pairs of pomalidomide analogs with and without $\mathrm{H}$-bond donor(s) immediately adjacent to the phthalimide ring. Plot showing pair-wise comparison of GFP degradation levels induced by the compound pairs at the same dose, ranging from $4.3 \mathrm{nM}$ to $20 \mu \mathrm{M}$. (F) Immunoblots for endogenous ZF proteins ZFP91 and IKZF3 in Jurkat cells treated with pomalidomide analogs (23-24) with and without a $\mathrm{H}$-bond donor $(\mathrm{NH})$ immediately attached to the phthalimide ring.

We aimed to identify exit vector modifications that confer the least off-target ZF degradation for PROTAC development. Towards this goal, we derived a degradation score for each pomalidomide analog, including pomalidomide-based PROTACs, by taking the sum of eGFP degradation values for the ZF degrons at multiple doses for each analog (Figure 3). Compounds with degradation scores close to zero induced the least ZF degradation, whereas compounds with the most negative scores induced the most ZF degradation (Figure 3). Analogs with piperazine and alkyne exit vectors predominated the group of compounds with degradation scores of 0 (Figure 3). Other exit vectors with minimal degradation scores include phenyl, diazaspiro[5.5]undecane, azetidine, pyrrolidine, 3,3-difluoropyrrolidine, morpholine, diazaspiro[3.3]heptane, and $N$-methylcyclohexylamine, as well as fluoro analogs of morpholine, $N$-protected piperazine, and $N$-protected diazaspiro[3.3]heptane (Figure 3B). From this study, we established two main rules for designing pomalidomide-based PROTACs to minimize off-target effects. First, exit vectors should predominantly have modifications on the C5 position. Second, none of the Hbond donors should be immediately adjacent to the phthalimide ring. Taken together, we have applied this systematic analysis of pomalidomide analogs and identified a collection of exit vectors to guide the development of pomalidomide-based PROTACS with minimal off-target ZF degradation. 

available under aCC-BY-NC-ND 4.0 International license.

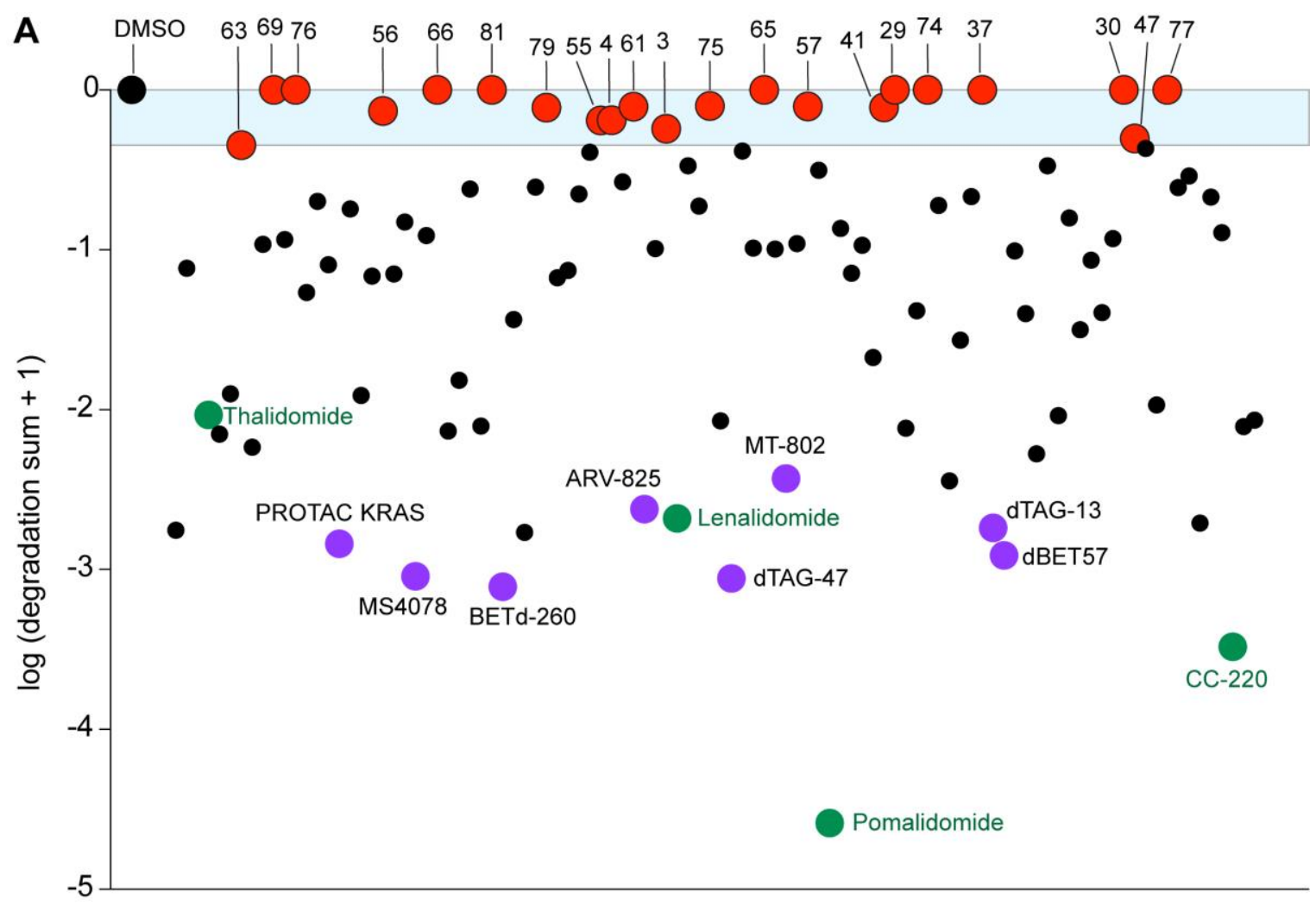

Pomalidomide analogs and PROTACs

B<smiles>O=C1CCC(N2C(=O)c3cccc(Br)c3C2=O)C(=O)N1</smiles><smiles>C1CN2CCN(C1)C2</smiles>

29<smiles>C1C2CN3CC2CN1C3</smiles>

30<smiles>CC#CC#[Tl]</smiles>

37<smiles>O=C1CCC2C(=O)NC(=O)N2C1=O</smiles>

$R^{2}=$<smiles>C1CCN2CCCC2C1</smiles><smiles>FC1(F)CC2CCCC2C1</smiles>

57

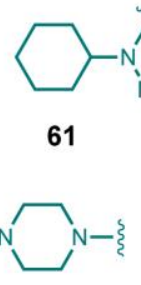

66

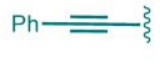

74<smiles>O=C1CCC2C(=O)c3cc(Cl)c(F)cc3C(=O)N2C(=O)N1</smiles><smiles>CN1CCN(C2CCCC2)CC1</smiles><smiles>O=[N+]1CCN(I)CC1</smiles><smiles>CN1CCC2(COC2)C1</smiles><smiles>CCN1CCC2(CC1)CC[N+](=O)CC2</smiles>

Figure 3. Degradation score as metric to nominate the IMiDs with reduced off-targets. A) Scatter dot plot showing ZF degradation scores [i.e. - Log(degradation sum + 1)] for individual pomalidomide analogs and pomalidomide-based PROTACs investigated in this study. Circles with red indicates the IMiD analogs with close to zero off-target ZF degradation and Circles filled with green are the parent class of IMiDs and Circles filled with purple indicate the reported PROTACs used in the study. See materials and methods section in supplementary information for details on computing the ZF degradation score. B) Structures of IMiD analogs with the least degradation (close to 0) are shown. 
Development of PROTACs with reduced off-target degradation propensities. As a proof-of-concept of our findings, we reengineered the potent reported ALK PROTAC (MS4078), ${ }^{33}$ which had a high level of off-target ZF degradation (Figure 1B-C), by altering the exit vectors on pomalidomide to reduce off-target ZF degradation while maintaining potency. We selected pomalidomide analogs with alkyne and piperazine exit vectors on the C5 positions, which had degradation scores close to zero (Figure 3) and included the C5 piperazine and 2,6diazaspiro[3.3]heptane with a C6 fluoro modification due to its near-zero ZF degradation score (Figures 2C and 3). We rationally designed and synthesized 12 new ALK PROTACs with different exit vectors, such as an alkyne (C4: dALK-1 and C5: dALK-2), piperazine with varying alkyl (dALK-3 to dALK-6) and acyl linkers (dALK-7 to 10), and diazaspiro[3.3]heptane (dALK-11 and dALK-12) by Sonogashira cross-coupling, reductive amination and amidation chemistry, respectively. To investigate the effect of the fluoro group on the on- and off-target propensities, we also synthesized the corresponding fluoro pairs for non-alkyne dALK PROTACs (dALK-4, 6, 8, 10, and 12) (Figure 4A).

We then performed the high-throughput imaging analysis of new ALK PROTACs to investigate the off-target profiles of these compounds. The reengineered ALK PROTAC with C5 alkyne exit vector (dALK-2) dramatically reduced the off-target effects of the original PROTAC MS4078, reducing the affinity for proteins such as ZNF517, ZNF654, ZNF276, ZNF653, and PATZ1 (Figure 4B). New ALK PROTACs with piperazine exit vectors with and without the fluoro group exhibited minimized off-target effects (Figure 4B).

We then performed cytotoxicity studies to investigate any enhancement in the on-target activity. One of the nonimide-based building blocks of parent ALK PROTAC MS4078 is ceritinib, a potent ALK inhibitor with an $\mathrm{EC}_{50}$ of 69.3 nM. However, its conversion to a degrader (MS4078) lowered its potency by 2.8-fold with high $\mathrm{EC}_{50}$ values (195.3 nM) (Figure 4C, and S8). Though introducing alkyne exit vectors reduced the off-target effects, its $\mathrm{EC}_{50}$ values increased, suggesting a reduction in the on-target potential likely due to the ineffective linker length to induce the proximity. We later performed the systematic linker length optimization for the piperazine and diazaspiro[3.3]heptane exit vectors and identified 7 potent ALK PROTACs with EC $_{50}$ values lower than that of MS4078. Strikingly, our ALK PROTACs with C5 piperazine exit vectors containing propyl/butyl amide linkers (dALK-7, 9, respectively) and a C5 diazaspiro[3.3]heptane linker containing propylamide (dALK-11) were found to be the most potent and the best PROTACs. Among these 7 ALK PROTACs, we identified two best-in-class 
bioRxiv preprint doi: https://doi.org/10.1101/2021.11.18.468552; this version posted November 20, 2021. The copyright holder for this preprint (which was not certified by peer review) is the author/funder, who has granted bioRxiv a license to display the preprint in perpetuity. It is made available under aCC-BY-NC-ND 4.0 International license.

A

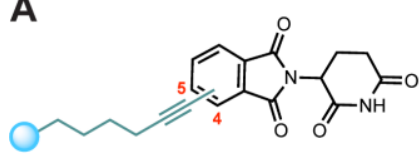
dALK-1 (C4) dALK-2 (C5)
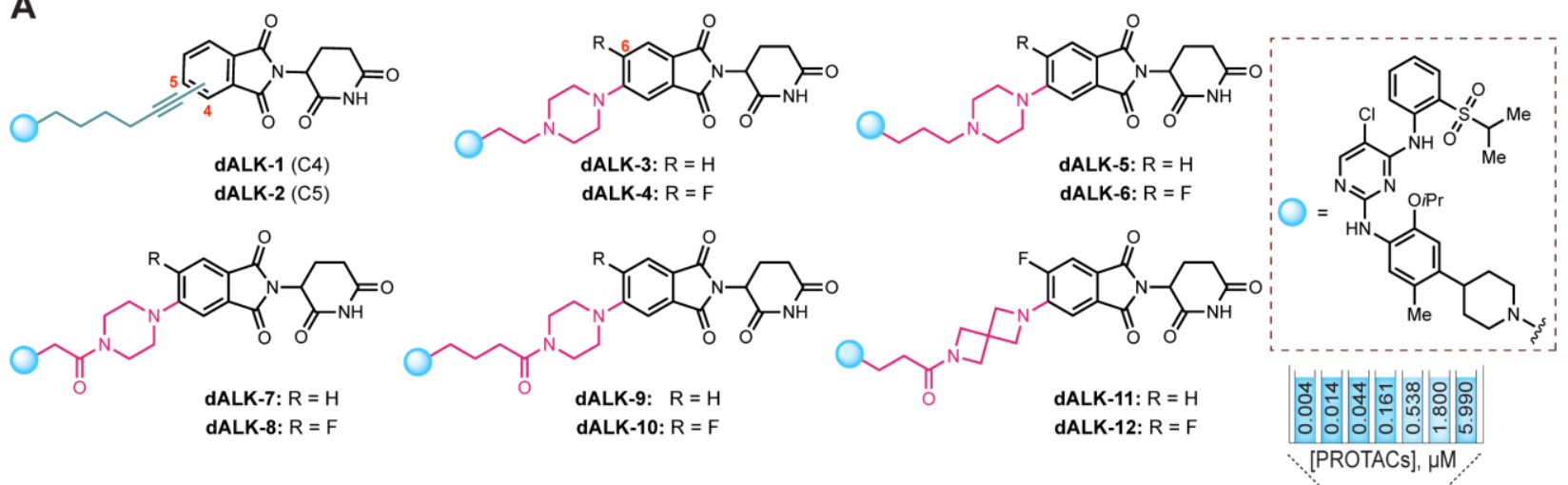

B

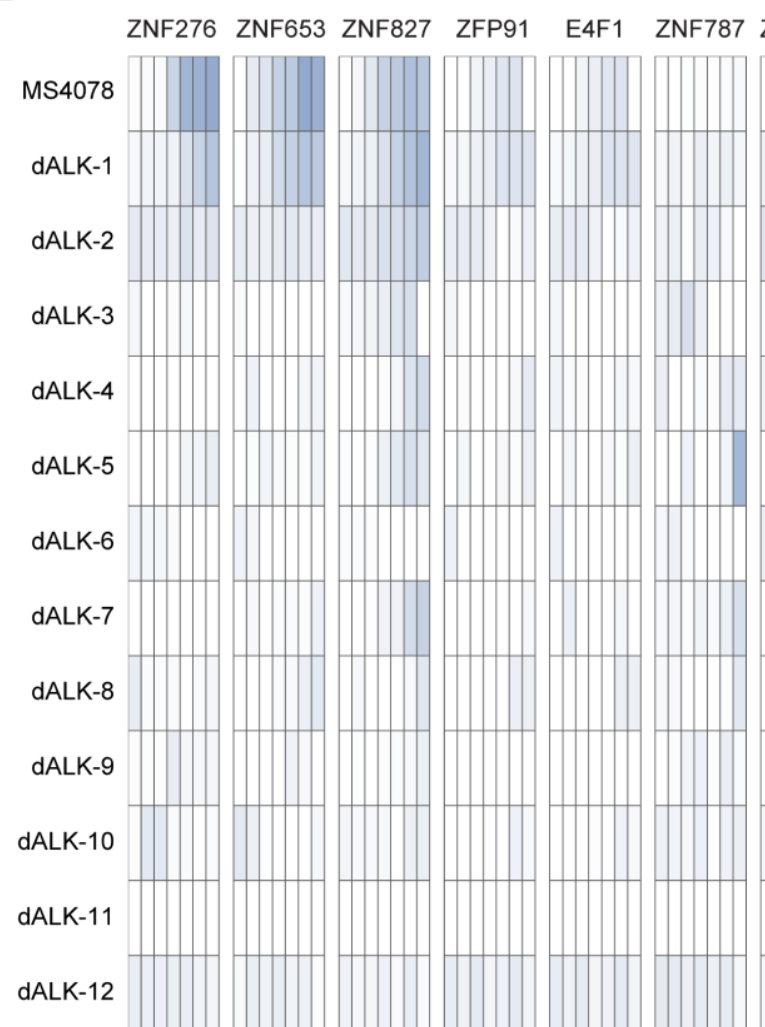

C

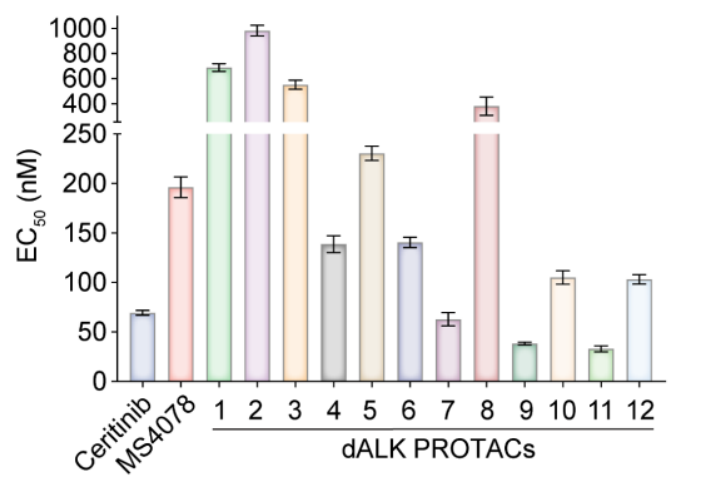

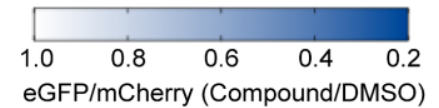

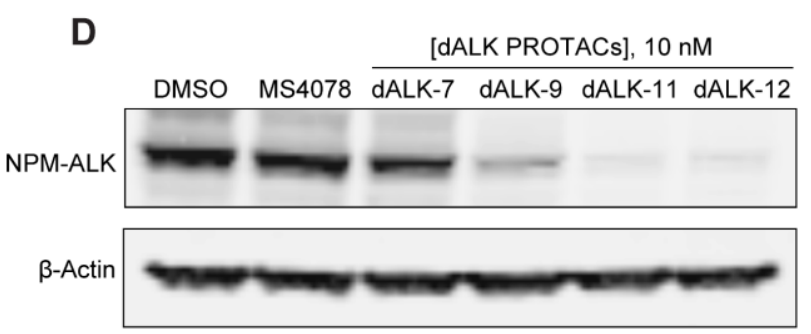

Figure 4. Reengineering of ALK PROTACs based on the new design principles. (A) Structures of rationally redesigned ALK PROTACs to minimize off-target ZF degradation and enhance the on-target potency. (B) Degradation of validated and pomalidomide-sensitive ZF degrons inside cells by redesigned ALK PROTACs 
in a dosing range of $4.3 \mathrm{nM}$ to $6 \mu \mathrm{M}$. (C) Determination of $\mathrm{EC}_{50}$ values $(\mathrm{nM})$ of SU-DH-L1 cells upon treatment with redesigned ALK PROTACs. (D) Immunoblots showing the degradation of ALK protein in SU-DH-L1 cells by the ALK PROTACs.

PROTACs with 1.8-2-fold higher potency than MS4078 and $\mathrm{EC}_{50}$ values of $32.8 \mathrm{nM}(\mathrm{dALK}-11)$ and $38.4 \mathrm{nM}$ (dALK-12), which renders them more effective in reducing the SU-DHL-1 cell viability (Figure 4C, and S8) by selectively degrading ALK protein. Furthermore, the immunoblot analysis of lysates from SU-DHL-1 cells revealed dALK-11 and dALK-12 as the potent degrader of ALK protein even at $10 \mathrm{nM}$ concentration (Figure 4D) corroborating with the cytotoxicity studies.

\section{CONCLUSIONS}

We have developed and validated a new and image-based high-throughput off-target profiling platform for the systematic evaluation of PROTACs that induce off-target degradation of ZF proteins, which play crucial roles in biology and disease progression. We leveraged this platform to identify the rules for designing pomalidomidebased PROTACs that minimize off-target degradation of ZF proteins by designing and testing a library of pomalidomide analogs. Here, the greatest reduction in off-target ZF degradation was achieved through modification of the exit vectors on the C5 position of the phthalimide ring via nucleophilic aromatic substitution ( $\left.S_{N} A r\right)$. Guided by these new designed principles, we provided a proof-of-concept by reengineering a reported ALK PROTAC, MS4078 for enhanced potency and reduced off-target degradation.

The new rules for pomalidomide-based PROTACs generated in this study can be readily applied to address the crucial need for PROTACs that do not indiscriminately degrade key ZF proteins. One drawback to our hereindeveloped high-throughput degradation assay is its limitation to the detection of the ZF degrons most sensitive to pomalidomide, but not the entire proteome. Furthermore, this assay ignores neosubstrates that can be degraded by these IMiD analogs. Regardless, the findings from this study offer opportunities to develop new and safer PROTACs as well as to improve on existent PROTACs with enhanced on-target potency. Our collection of synthetic pomalidomide derivatives with varied exit vectors that affect minimal off-target ZF degradation can be widely adopted for the generation of safer and clinically relevant PROTACs. Notably, recently reported 
PROTACs in advanced clinical trials at Arvinas, Inc. have the similar exit vector with piperazine, which agreed with our findings of minimal ZF degradation. Conclusively, the case study herein provides further confidence and validation for the potential to apply exit vectors discovered in this work for the benefit of clinical applications and to the PROTAC community at large.

\section{ACKNOWLEDGEMENTS}

We thank Patrick Byrne (Broad Institute) for assistance with automated imaging screening experiments. This work was supported by DARPA (N66001-17-2-4055) and NIH (R01 GM137606 and R01 GM132825 to A.C.; R01 EB031172, R01 EB027793, and R35 GM118062 to D.R.L. J.A.M.M. is a Ruth L. Kirchstein National Research Service Award Postdoctoral Fellow (F32 GM133088). D.R.L. and B. L. E. are investigators of the Howard Hughes Medical Institute.

\section{COMPETING FINANCIAL INTERESTS}

Broad Institute has filed a patent application including the work described herein. 
bioRxiv preprint doi: https://doi.org/10.1101/2021.11.18.468552; this version posted November $20,2021$. The copyright holder for this preprint (which was not certified by peer review) is the author/funder, who has granted bioRxiv a license to display the preprint in perpetuity. It is made available under aCC-BY-NC-ND 4.0 International license.

\section{REFERENCES}

1. Chamberlain, P. P.; Cathers, B. E., Cereblon modulators: Low molecular weight inducers of protein degradation. Drug Discov Today Technol 2019, 31, 29-34.

2. Kozicka, Z.; Thomä, N. H., Haven't got a glue: Protein surface variation for the design of molecular glue degraders. Cell Chem Biol 2021, 28 (7), 1032-1047.

3. Finley, D., Recognition and processing of ubiquitin-protein conjugates by the proteasome. Annu Rev Biochem 2009, 78, 477-513.

4. Ito, T.; Ando, H.; Suzuki, T.; Ogura, T.; Hotta, K.; Imamura, Y.; Yamaguchi, Y.; Handa, H., Identification of a primary target of thalidomide teratogenicity. Science (New York, N.Y.) 2010, 327 (5971), 1345-50.

5. Krönke, J.; Udeshi, N. D.; Narla, A.; Grauman, P.; Hurst, S. N.; McConkey, M.; Svinkina, T.; Heckl, D.; Comer, E.; Li, X.; Ciarlo, C.; Hartman, E.; Munshi, N.; Schenone, M.; Schreiber, S. L.; Carr, S. A.; Ebert, B. L., Lenalidomide causes selective degradation of IKZF1 and IKZF3 in multiple myeloma cells. Science (New York, N.Y.) 2014, 343 (6168), 301-5.

6. Lu, G.; Middleton, R. E.; Sun, H.; Naniong, M.; Ott, C. J.; Mitsiades, C. S.; Wong, K. K.; Bradner, J. E.; Kaelin, W. G., Jr., The myeloma drug lenalidomide promotes the cereblon-dependent destruction of Ikaros proteins. Science (New York, N.Y.) 2014, 343 (6168), 305-9.

7. Chamberlain, P. P.; Hamann, L. G., Development of targeted protein degradation therapeutics. Nat Chem Biol 2019, 15 (10), 937-944.

8. Sakamoto, K. M.; Kim, K. B.; Kumagai, A.; Mercurio, F.; Crews, C. M.; Deshaies, R. J., Protacs: chimeric molecules that target proteins to the Skp1-Cullin-F box complex for ubiquitination and degradation. Proc Natl Acad Sci U S A 2001, 98 (15), 8554-9.

9. Winter, G. E.; Buckley, D. L.; Paulk, J.; Roberts, J. M.; Souza, A.; Dhe-Paganon, S.; Bradner, J. E., Drug Development. Phthalimide conjugation as a strategy for in vivo target protein degradation. Science (New York, N.Y.) 2015, 348 (6241), 1376-81.

10. Jiang, B.; Wang, E. S.; Donovan, K. A.; Liang, Y.; Fischer, E. S.; Zhang, T.; Gray, N. S., Development of Dual and Selective Degraders of Cyclin-Dependent Kinases 4 and 6. Angew Chem Int Ed 2019, 58 (19), 6321-6326.

11. Teng, M.; Jiang, J.; He, Z.; Kwiatkowski, N. P.; Donovan, K. A.; Mills, C. E.; Victor, C.; Hatcher, J. M.; Fischer, E. S.; Sorger, P. K.; Zhang, T.; Gray, N. S., Development of CDK2 and CDK5 Dual Degrader TMX-2172. Angew Chem Int Ed 2020, 59 (33), 13865-13870.

12. Matyskiela, M. E.; Lu, G.; Ito, T.; Pagarigan, B.; Lu, C. C.; Miller, K.; Fang, W.; Wang, N. Y.; Nguyen, D.; Houston, J.; Carmel, G.; Tran, T.; Riley, M.; Nosaka, L.; Lander, G. C.; Gaidarova, S.; Xu, S.; Ruchelman, A. L.; Handa, H.; Carmichael, J.; Daniel, T. O.; Cathers, B. E.; Lopez-Girona, A.; Chamberlain, P. P., A novel cereblon modulator recruits GSPT1 to the CRL4(CRBN) ubiquitin ligase. Nature 2016, 535 (7611), 252-7.

13. Petzold, G.; Fischer, E. S.; Thomä, N. H., Structural basis of lenalidomide-induced CK1a degradation by the CRL4(CRBN) ubiquitin ligase. Nature 2016, 532 (7597), 127-30.

14. Krönke, J.; Fink, E. C.; Hollenbach, P. W.; MacBeth, K. J.; Hurst, S. N.; Udeshi, N. D.; Chamberlain, P. P.; Mani, D. R.; Man, H. W.; Gandhi, A. K.; Svinkina, T.; Schneider, R. K.; McConkey, M.; Järås, M.; Griffiths, E.; Wetzler, M.; Bullinger, L.; Cathers, B. E.; Carr, S. A.; Chopra, R.; Ebert, B. L., Lenalidomide induces ubiquitination and degradation of CK1 $\alpha$ in del(5q) MDS. Nature 2015, 523 (7559), 183-188.

15. Wang, A.; Ding, L.; Wu, Z.; Ding, R.; Teng, X. L.; Wang, F.; Hu, Z.; Chen, L.; Yu, X.; Zou, Q., ZFP91 is required for the maintenance of regulatory $T$ cell homeostasis and function. $J$ Exp Med 2021, 218 (2).

16. Fu, M.; Blackshear, P. J., RNA-binding proteins in immune regulation: a focus on $\mathrm{CCCH}$ zinc finger proteins. Nat Rev Immunol 2017, 17 (2), 130-143.

17. Cassandri, M.; Smirnov, A.; Novelli, F.; Pitolli, C.; Agostini, M.; Malewicz, M.; Melino, G.; Raschellà, G., Zincfinger proteins in health and disease. Cell Death Discov 2017, 3, 17071.

18. Wang, E. S.; Verano, A. L.; Nowak, R. P.; Yuan, J. C.; Donovan, K. A.; Eleuteri, N. A.; Yue, H.; Ngo, K. H.; Lizotte, P. H.; Gokhale, P. C.; Gray, N. S.; Fischer, E. S., Acute pharmacological degradation of Helios destabilizes regulatory T cells. Nat Chem Biol 2021, 17 (6), 711-717.

19. Ito, T.; Ando, H.; Handa, H., Teratogenic effects of thalidomide: molecular mechanisms. Cell Mol Life Sci 2011, 68 (9), 1569-79. 
bioRxiv preprint doi: https://doi.org/10.1101/2021.11.18.468552; this version posted November 20,2021 . The copyright holder for this preprint (which was not certified by peer review) is the author/funder, who has granted bioRxiv a license to display the preprint in perpetuity. It is made available under aCC-BY-NC-ND 4.0 International license.

20. Therapontos, C.; Erskine, L.; Gardner, E. R.; Figg, W. D.; Vargesson, N., Thalidomide induces limb defects by preventing angiogenic outgrowth during early limb formation. Proc Natl Acad Sci U S A 2009, 106 (21), 8573-8.

21. Donovan, K. A.; An, J.; Nowak, R. P.; Yuan, J. C.; Fink, E. C.; Berry, B. C.; Ebert, B. L.; Fischer, E. S., Thalidomide promotes degradation of SALL4, a transcription factor implicated in Duane Radial Ray syndrome. Elife 2018, 7.

22. Matyskiela, M. E.; Couto, S.; Zheng, X.; Lu, G.; Hui, J.; Stamp, K.; Drew, C.; Ren, Y.; Wang, M.; Carpenter, A.; Lee, C. W.; Clayton, T.; Fang, W.; Lu, C. C.; Riley, M.; Abdubek, P.; Blease, K.; Hartke, J.; Kumar, G.; Vessey, R.; Rolfe, M.; Hamann, L. G.; Chamberlain, P. P., SALL4 mediates teratogenicity as a thalidomidedependent cereblon substrate. Nat Chem Biol 2018, 14 (10), 981-987.

23. Mullard, A., Targeted protein degraders crowd into the clinic. Nature Rev Drug Discov 2021, 20 (4), $247-250$.

24. Nguyen, P. A.; Born, D. A.; Deaton, A. M.; Nioi, P.; Ward, L. D., Phenotypes associated with genes encoding drug targets are predictive of clinical trial side effects. Nat Commun 2019, 10 (1), 1579.

25. Deaton, A. M.; Fan, F.; Zhang, W.; Nguyen, P. A.; Ward, L. D.; Nioi, P., Rationalizing Secondary Pharmacology Screening Using Human Genetic and Pharmacological Evidence. Toxicol Sci 2019, 167 (2), 593-603.

26. Zhang, A. X.; Cassidy, K.; Dahl, G.; Moreau, K.; Pachl, F.; Zuhl, A. M., The Vital Role of Proteomics in Characterizing Novel Protein Degraders. SLAS Discov 2021, 26 (4), 518-523.

27. Beveridge, R.; Kessler, D.; Rumpel, K.; Ettmayer, P.; Meinhart, A.; Clausen, T., Native Mass Spectrometry Can Effectively Predict PROTAC Efficacy. ACS Cent Sci 2020, 6 (7), 1223-1230.

28. Grandi, P.; Bantscheff, M., Advanced proteomics approaches to unravel protein homeostasis. Drug Discov Today Technol 2019, 31, 99-108.

29. Liu, X.; Zhang, Y.; Ward, L. D.; Yan, Q.; Bohnuud, T.; Hernandez, R.; Lao, S.; Yuan, J.; Fan, F., A proteomic platform to identify off-target proteins associated with therapeutic modalities that induce protein degradation or gene silencing. Sci Rep 2021, 11 (1), 15856.

30. Reinders, J.; Lewandrowski, U.; Moebius, J.; Wagner, Y.; Sickmann, A., Challenges in mass spectrometrybased proteomics. Proteomics 2004, 4 (12), 3686-703.

31. Donovan, K. A.; Ferguson, F. M.; Bushman, J. W.; Eleuteri, N. A.; Bhunia, D.; Ryu, S.; Tan, L.; Shi, K.; Yue, H.; Liu, X.; Dobrovolsky, D.; Jiang, B.; Wang, J.; Hao, M.; You, I.; Teng, M.; Liang, Y.; Hatcher, J.; Li, Z.; Manz, T. D.; Groendyke, B.; Hu, W.; Nam, Y.; Sengupta, S.; Cho, H.; Shin, I.; Agius, M. P.; Ghobrial, I. M.; Ma, M. W.; Che, J.; Buhrlage, S. J.; Sim, T.; Gray, N. S.; Fischer, E. S., Mapping the Degradable Kinome Provides a Resource for Expedited Degrader Development. Cell 2020, 183 (6), 1714-1731.e10.

32. Sievers, Q. L.; Petzold, G.; Bunker, R. D.; Renneville, A.; Słabicki, M.; Liddicoat, B. J.; Abdulrahman, W.; Mikkelsen, T.; Ebert, B. L.; Thomä, N. H., Defining the human $\mathrm{C} 2 \mathrm{H} 2$ zinc finger degrome targeted by thalidomide analogs through CRBN. Science 2018, 362 (6414), eaat0572.

33. Zhang, C.; Han, X.-R.; Yang, X.; Jiang, B.; Liu, J.; Xiong, Y.; Jin, J., Proteolysis Targeting Chimeras (PROTACs) of Anaplastic Lymphoma Kinase (ALK). Eur J Med Chem 2018, 151, 304-314.

34. Nabet, B.; Roberts, J. M.; Buckley, D. L.; Paulk, J.; Dastjerdi, S.; Yang, A.; Leggett, A. L.; Erb, M. A.; Lawlor, M. A.; Souza, A.; Scott, T. G.; Vittori, S.; Perry, J. A.; Qi, J.; Winter, G. E.; Wong, K.-K.; Gray, N. S.; Bradner, J. E., The dTAG system for immediate and target-specific protein degradation. Nat Chem Biol 2018, 14 (5), 431-441. 